tient stets gesund gewesen sein. Am 25. Juli 1902 kam er das erste $\mathrm{Mal}$ in die Poliklinik, weil das erkrankte linke Ohr seit einigen Tagen stärker eiterte, und bisweilen Schmerzen aufgetreten waren. An diesem Tage wurde ein sehr grosser Polyp entfernt, die auffallend starke Blutung durch Tamponade gestillt, Patient zum nächsten Tage wiederbestellt; er kam jedoch erst acht Tage später, und wurden ihm noch einmal kleinere Granulationen entfernt.

Am 21. August - Patient hatte in der Zwischenzeit nur noch einmal die Poliklinik besucht - stellten wir bei ihm eine complete, linksseitige Facialislähmung fest; auf Befragen gab der Patient auch an, er bemerke seit sechs Tagen, dass sein Gesicht links schief sei, auch habe er häufiger in der letzten Zeit Kopfschmerzen gehabt, allerdings mehr auf der rechten Kopfseite. Am Tage vorher will der Patient einen starken Schüttelfrost gehabt haben. Dem Patienten wurde angerathen, sich in die Klinik aufnehmen zu lassen, er that dies jedoch erst am 25. August. An diesem Tage war der Status im linken Ohr folgender: Gehörgang sehr weit, sehr viel fötides Sekret, in der Tiefe Gra-

III. Aus Dr. J. Herzfeld's Klinik für Hals-, Nasen- und Ohrenkrankheiten in Berlin.

\section{Kleinhirnabszess mit plötzlicher Lähmung des Respirationscentrums.')}

Von Dr. H. Fliess, Assistenzarzt.

O. R., 30 Jahre alt, leidet seit Kindheit im Anschluss an Masern an Ausfluss aus dem linken $\mathrm{Ohr}$, der nie aufgehört hat; sonst will $\mathrm{Pa}$ - nulationen, Trommelfell fehlt vollkommen.

Hörprüfung: Flüstern links nicht vernommen.

Stimmgabelprüfung: c vom Scheitel nach rechts, c per Luft nicht vernommen, hohe Töne $\left(\right.$ fis $\left._{4}\right)$ bedeutend herabgesetzt.

Der Warzenfortsatz nicht aufgetrieben, nicht schmerzhaft. Die Drehungen des Kopfes um alle Axen frei. Sensibilität überall erhalten. Keine Kopfschmerzen, kein Fieber, Puls 80.

Aber schon am nächsten Tage hatte sich das Bild wesentlich verändert. In der Nacht war 2-3mal Erbrechen aufgetreten, die Drehungen des Kopfes sind nach vorn und hinten schmerzhaft, geringe Nackensteifigkeit. Das Beklopfen des Kopfes fast überall schmerzhaft. Patient ist nicht bei klarem Bewusstsein, steht z. B. auf, um das Bett zu machen, will sich anziehen und nach Hause gehen. Temperatur 36,8, Puls 50.

Im Laufe des Tages nahm die Nackensteifigkeit bedeutend zu. Die Pupillen sind mittel und gleichweit. Cornealreflex links erloschen, rechts erhalten. Die Pupillen in mässiger Myosis reagiren nur mit einem kaum merklichen Zusammenziehen. Die Papillen sind blass, scharf umgrenzt, die Venen links voller als rechts, besonders die untere. Die Sensibilität ist links bedeutend herabgesetzt. Bauch- und Cremasterreflex nicht auszulösen, Patellarreflex beiderseits deutlich, ebenso Babinski. Die linke obere Extremität fällt, wenn man sie hochhebt, schlaff herab.

Dieses Krankheitsbild, welches sich an diesem Tage hintereinander entwickelt hatte, liess wohl einen Abszess vermuthen, und zwar wies die Facialislähmung, die mangelnde Hörfähigkeit und vor allem die Nackensteifigkeit am meisten auf das Kleinhirn hin. Bei der Unsicherheit der Diagnose sollten nun zuerst die Mittelohrräume freigelegt werden in der Hoffnung, von hier aus vielleicht einen Fingerzeig für das weitere Vorgehen zu finden. Aber schon nach wenigen Meisselschlägen - die Operation wurde in Aethernarkose gemacht - setzte die Athmung aus, sodass die Operation unterbrochen werden musste, dabei blieb der Puls fortgesetzt leidlich gut. Obwohl zwei Stunden lang künstliche Athmung unterhalten, Sauerstoffinhalationen angewandt wurden, trat der Exitus ein.

Die Autopsie deckte in der That einen Abszess an der unteren Fläche des Kleinhirns auf, der den vorderen äusseren Quadranten einnimmt, wenn man sich die Kleinhirnfläche in vier Quadranten zerlegt denkt. Die Abszesshöhle beträgt $3 \frac{1}{2} \mathrm{~cm}$ im Quer-, $3 \mathrm{~cm}$ im Sagittal-, ca. $2 \mathrm{~cm}$ im Frontaldurchmesser. Die innere Wand der Abszesshöhle ist stark erweicht und zertrümmert. Das abgerissene Stück der Abszesshöhle (äussere Wand der Kleinhirnhemisphäre) ist in der vorderen Hïlfte vollständig vereitert, und dieser Theil ist mit dem Felsenbein verwachsen, und zwar direkt mit der den Cotugno'schen Sack bedeckenden Duralpartie. Die Austrittstellen des Nervus facialis und Acusticus waren nicht vereitert. - Man darf wohl mit Sicherheit annehmen, dass der Eiter auf dem Wege des Aquaeductus vestibuli die hintere Schädelgrube erreicht und den Abszess veranlasst hat

Derartige otogene Kleinhirnabszesse, die auf dem Wege des Aquaeductus vestibuli entstanden sind, hat $\mathrm{J}_{\text {anse }} \mathrm{n}^{1}$ ) zuerst in seiner Arbeit: „Zur Kenntniss der durch Labyrintheiterung induzirten, tiefen extraduralen Abszesse in der hinteren Schädelgrube" beschrieben, und giebt er als Symptomenbild Folgendes an: „Durch den Eiterheerd werden meningitische Reizerscheinungen ausgelöst, ohne dass bereits eine eitrige Arachnitis besteht. Unter dem Bilde einer schweren Krankheit mit hohem Fieber, trockener, belegter Zunge sahen wir heftigen Kopfschmerz, öfters Schwindel, Erbrechen, leichte Benommenheit, Unruhe, das Krankheitsbild sehr bedrohlich gestalten, welches sich aber erst nach 12-14 Tagen zu einer unzweideutigen, rasch terminalen Meningitis entwickelte. Der Tod war 18 Tage nach Einleitung der Labyrintheiterung durch Steigbügelluxation beim Ausschaben erfolgt."

Dass aber diese stürmischen Symptome nicht immer nöthig sind, beschreibt Schulze ) aus der Hallenser Ohrenklinik in einer Arbeit "Zur Kenntniss des Empyems des Sacculus endolymphaticus“.
1) Nach einem in der Berliner otologischen Gesellschaft mit Demonstration gehaltenen Vortrag.
1) Archiv für Ohrenheilkunde Bd. XXXV.

2) Archiv für Ohrenheilkunde Bd. LVII. 
In diesem Falle traten $2 \frac{1}{2}$ Monate nach der Totalaufmeisselung plötzlich sehr heftiger Kopfschmerz, Schwindel und Erbrechen ohne Temperaturerhöhung auf. Diese Erscheinungen gingen in zwei Tagen gänzlich zurück, wiederholten sich nach 14 Tagen und führten in einem Tage zum Exitus. Die Sektion ergab eine Meningitis, einen Kleinhirnabszess und ein Empyem des Sacculus endolymphaticus.

Auch unser Fall zeigt, wie symptomenlos auf dem Wege des Aquaeductus vestibuli und des Sacculus endolymphaticus ein solcher Abszess entstehen kann. Leider hat derselbe, wie es auch öfters in der Litteratur beschrieben wird, bis zum letzten Tage gar keine Erscheinungen gemacht, da bei dem Sitz des Abszesses der Patient wohl hätte gerettet werden können.

Besonders bemerkenswerth bei diesem Falle ist die Erscheinung, dass der Puls noch zwei Stunden lang relativ gut war, während die Athmung nur noch künstlich unterhalten wurde.

Die Ursache dieser Erscheinung ist der abnorm gesteigerte Hirndruck.

Aehnliche Fülle mit Erlöschen der Respiration vor dem Ausbleiben des Pulses werden einige Male beschrieben. So giebt Sir Dyce Duckworth') in einem auf dem Moskauer Congress gehaltenen Vortrage die genaue Krankengeschichte von vier Fällen; er beschreibt einen

1) Kleinhirnabszess nach Otitis med.,

2) Abszess im Temporallappen nach Otitis med.,

3) chronischen Hirnabszess im Temporallappen,

4) Fall von traumatischer, subcortikaler Blutung an der Vereinigungsstelle des Temporal-, Occipital- und Parietallappens des Grosshirns.

Bei dreien dieser Fälle trat in der Narkose die Respirationslähmung ein, bei einem spontan. Ferner erwähnt Duckworth zwei Fälle von Maceven, bei denen nach stundenlang fortgesetzter künstlicher Athmung die Respiration sich wiedereinstellte.

Ueber einen Fall von Respirationslähmung bei einem Kleinhirntumor, bei welchem tagelang künstliche Athmung unterhalten wurde, berichten Russel und Jackson ${ }^{2}$ ).

In der Prager medizinischen Wochenschrift veröffentlicht Dr. Hoffer ${ }^{3}$ ) einen Fall von ehronischem Kleinhirnabszess. Der Puls schlug noch $1 \frac{1}{2}$ Stunden ungestört fort, während die Athmung schon endgiltig aufgehört hatte.

A. E. Barker beschreibt in dem British medical Journal einen Kleinhirnabszess mit plötzlicher Lăhmung des Respirationscentrums bei einem 14jährigen Mädchen. Die Athmung hörte beim Aufheben auf den Operationstisch plötzlich auf. Obwohl der Eiter aus dem Kleinhirn entleert wurde, starb das Mädchen. Der Puls schlug noch 2 Stunden 35 Minuten lang, nachdem die natürliche Athmung aufgehört hatte. Künstliche Respiration wurde 2 Stunden lang gemacht.

Maceven erwähnt in seinem Werk Die infektiös-eitrigen Erkrankungen des Hirns und Rückenmarkes" mehrmals diese Complikation und berichtet über einen Hirnabszess, bei dem die künstliche Athmung ohne Trepanation 24 Stunden lang unterhalten wurde. Maceven will diese Erscheinung nur bei Affektionen der hinteren Schädelgrube kennen.

Wie schon erwähnt, ist die Ursache in dem abnorm gesteigerten Hirndruck zu suchen. Das vergrösserte Kleinhirn verschliesst mechanisch den Aquaeductus sylvii, es kommt hierdurch zur rapiden Dilatation der Seitenventrikel.

Maceven warnt vor dem Gebrauch von Anästhetica bei solchen Fällen, weil das Hirn oft ödematös ist, die erweiterten Seitenventrikel mit seröser Flüssigkeit gefüllt sind, und schon eine geringe Zunahme der serösen Flüssigkeit ein Aussetzen der Respiration veranlassen kann.

In der That treten die meisten Asphyxien in Narkose auf; wahrscheinlich erliegen die durch den Hirndruck geschädigten Ganglienzellen leichter der Giftwirkung des Narcoticums.

Nach der Annahme Duckworth's beweist die Lähmung des Respirationscentrums eine grössere Empfindlichkeit desselben gegen: über den Regulirungscentren der Herzaktion. Diese Annahme steht mit der Thatsache im Einklang, dass die Hirncentren schädigenden Einflüssen, speziell dem Hirndruck und den Giften, eine gesetzmässig abgestufte, graduell verschiedene Widerstandsfahigkeit entgegensetzen Bei der Narkose z. B. erlischt zuerst die intellektuelle Sphäre, dann folgen Sinneswahrnehmungen, motorische Coordination, Reflexe, schliesslich die automatischen Centren der Athmung und des Kreislaufes.

Wenn einmal die Athmung aufgehört hat, so könnte man in geeigneten Fällen dieser Art die Trepanation bei künstlicher Athmung versuchen; oder aber die Lumbalpunktion könnte eine zeitweise Verringerung des Hirndruckes and Wiederkehr der Respiration für die Daner der Operation herbeiführen. Andererseits ist es aber fraglich, ob die Lumbalpunktion auszuführen sein wird. Ist die Theorie richtig,

1) Some cases of cerebral disease, in which the function of respiration entirely ceases for some hours before that of the circulation. By Sir Dyce Duckworth. The Edinburgh medical Journal, February 1898.

2) Nothnagel's Pathologie und Therapie Bd. IX.

3) Prager medizinische Wochenschrift No. XX. dass der vierte Ventrikel zusammengedrückt, und die Seitenventrikel sehr ausgebreitet sind, so kommt es zum passiven Verschlusse der Ventrikelöffnungen. Die Punktion wird negativ ausfallen.

In unserem Falle bestanden übrigens eine grössere Ausdehnung und Füllung der Ventrikel nicht. 